ACCEPTED MANUSCRIPT

\title{
Influence of moisture absorption on electrical properties and charge dynamics of polyethylene silica-based nanocomposite
}

To cite this article before publication: Yan Wang et al $2018 \mathrm{~J}$. Phys. D: Appl. Phys. in press https://doi.org/10.1088/1361-6463/aadb7b

\section{Manuscript version: Accepted Manuscript}

Accepted Manuscript is "the version of the article accepted for publication including all changes made as a result of the peer review process, and which may also include the addition to the article by IOP Publishing of a header, an article ID, a cover sheet and/or an 'Accepted

Manuscript' watermark, but excluding any other editing, typesetting or other changes made by IOP Publishing and/or its licensors"

This Accepted Manuscript is @ 2018 IOP Publishing Ltd.

During the embargo period (the 12 month period from the publication of the Version of Record of this article), the Accepted Manuscript is fully protected by copyright and cannot be reused or reposted elsewhere.

As the Version of Record of this article is going to be / has been published on a subscription basis, this Accepted Manuscript is available for reuse under a CC BY-NC-ND 3.0 licence after the 12 month embargo period.

After the embargo period, everyone is permitted to use copy and redistribute this article for non-commercial purposes only, provided that they adhere to all the terms of the licence https://creativecommons.org/licences/by-nc-nd/3.0

Although reasonable endeavours have been taken to obtain all necessary permissions from third parties to include their copyrighted content within this article, their full citation and copyright line may not be present in this Accepted Manuscript version. Before using any content from this article, please refer to the Version of Record on IOPscience once published for full citation and copyright details, as permissions will likely be required. All third party content is fully copyright protected, unless specifically stated otherwise in the figure caption in the Version of Record.

View the article online for updates and enhancements. 


\title{
Influence of Moisture Absorption on Electrical Properties and Charge Dynamics of Polyethylene Silica-Based Nanocomposites
}

\author{
Yan Wang, Dayuan Qiang, Fuad N. F. Alhabill, Zhiqiang Xu, George Chen and Alun \\ Vaughan
}

${ }^{1}$ The Tony Davies High Voltage Laboratory, University of Southampton

E-mail: yw14g13@soton.ac.uk

Received xxxxxx

Accepted for publication $\mathrm{xxxxxx}$

Published xxxxxx

\begin{abstract}
The use of nanocomposites as dielectric materials is expected to lead to improved electrical performance. However, recent research has shown that moisture absorption can cause a deterioration in the electrical performance of nanocomposites. Although it is generally accepted that hydroxyl groups attached to nanoparticle surfaces are the main cause of moisture absorption, the impact of this absorption on the electrical properties of nanocomposites is still not fully understand. In this paper, a series of measurements, including thermogravimetric analysis, DC breakdown, surface potential decay and space charge, are conducted with the aim of determining the impact of moisture absorption on the electrical properties of polyethylene/silica nanocomposites. The results show that the loading ratio of nanosilica and the humidity of the conditioning environment determine the amount of absorbed moisture. According to the Zhuravlev model, the main contribution to the deterioration in electrical properties of nanocomposites comes from the large amount of moisture absorbed in multilayer form. It is found that the loading ratio of nanosilica is the most significant factor in reducing DC breakdown strength.
\end{abstract}

Keywords: nanocomposite; moisture absorption; polyethylene; nanosilica; DC breakdown; charge dynamics

\section{Introduction}

The concept of a nanodielectric was first proposed in 1994 [1], and such materials have attracted considerable attention during the following two decades. A number of interesting results have been obtained showing the desirable electrical properties of nanodielectrics. For example, magnesium oxide/low-density polyethylene (LDPE) nanocomposites exhibit improved DC breakdown strength and effectively suppressed space-charge formation compared with neat LDPE [2]. However, it has been found that absorption of moisture by the incorporated nanoparticles can lead to a deterioration in the electrical properties of nanocomposites.
This moisture absorption is generally related to the aspect ratio and surface chemical groups of the nanoparticles $[3,4]$. For instance, nanosilica-based cross-linked polyethylene (XLPE) nanocomposites are able to absorb a greater amount of moisture than the host XLPE owing to the hydroxyl groups attached to the surface of the nanosilica particles, leading to a decrease in AC breakdown strength, an increase in space-charge formation, and a reduction in water tree aging of wetted specimens [5]. The results for polyethylene/nanosilica indicate that moisture absorption can lead to increases in permittivity and loss tangent [4]. For polyimide/aluminium oxide nanocomposites, a reduction in breakdown strength and facilitation of surface potential decay due to moisture absorption have been reported [6]. 
Ethylene-vinyl acetate incorporated with organically modified nanofiller clay showed worsened electrical properties owing to moisture absorption dependent on the aspect ratio of the nanofiller, and it was found that a higher aspect ratio led to greater moisture absorption [3]. Moreover, the aspect ratio of the nanoparticles strongly affects the stress distribution within the matrix reinforcement, and this stress can further affect the diffusion rate of absorbed moisture [79].

Present understanding of moisture absorption behaviour in nanocomposites is incomplete because, although moisture can be present in several forms on the nanoparticle surface, this has not been considered carefully in previous investigations. Moreover, although there have been many experimental and theoretical investigations of the impact of moisture absorption on the electrical properties of nanocomposites, a reasonable description that links moisture absorption with electrical properties and charge dynamics is still lacking. For example, percolation theory has been used to model and explain the function of the water shell in charge dynamics [5,10], and the results obtained can be used to estimate the water shell size in terms of nanoparticle loading ratios, but this approach cannot deal with the impact of different forms of moisture on charge transport in nanocomposites from the perspective of the different chemical groups attached to the nanoparticle surface.

In this paper, the influence of moisture absorption on the electrical properties of blended polyethylene/silica nanocomposites containing untreated trimethoxy(propyl)silane-treated nanosilica is studied. In this material system, the nanosilica is more hygroscopic than the polyethylene. Therefore, moisture absorption is primarily by the nanoparticles rather than the host polymer. The moisture absorbed is measured by weighing specimens and finding the change in mass and by thermogravimetric analysis (TGA). Experiments based on DC electric fields, investigating space charge, surface potential decay and breakdown strength, are carried out. The surface chemical groups of nanosilica and their influence on absorbed moisture are discussed in terms of the Zhuravlev model [11]. The purpose of this research is to reveal the forms in which moisture is absorbed and study their impact on the DC breakdown strength and charge dynamics associated with hydroxyl groups attached to the nanosilica surface.

\section{Surface Chemical Groups of Nanosilica}

Many species of nanoparticles have been reported to cause moisture absorption, including $\mathrm{SiO}_{2}, \mathrm{Si}_{3} \mathrm{~N}_{4}, \mathrm{MgO}$, $\mathrm{Al}_{2} \mathrm{O}_{3}, \mathrm{BN}$ and nanoclays [3,6,12-16]. The moisture uptake for each specific nanoparticle may be different, but in all cases the absorption process can occur either before or after specimen manufacture when the nanoparticle or nanocomposite is exposed to a humid environment.
Nanosilica has frequently been used in research on nanodielectrics because it is widely available in a range of sizes, from tens to hundreds of nanometres, and also because of a number of favourable properties, such as a relatively high breakdown strength and electrical resistivity [14]. In addition, from an economic perspective, it can be produced at low cost by flame hydrolysis or polymerisation. However, these production methods also make nanosilica hydrophilic. To obtain spherical nanosilica, both flame hydrolysis and polymerisation processes will hydroxylate the surface of the silica, generating $\mathrm{Si}-\mathrm{OH}$ (silanol) groups on this surface $[11,14]$. The hydroxyl $(\mathrm{OH})$ group is covalently bonded with $\mathrm{Si}$ atoms on the surface and inside the particles. It is known that the presence of $\mathrm{OH}$ groups on the surface of nanosilica particles can change the surface properties, depending on the concentration of these groups [11]

Besides causing moisture absorption, the $\mathrm{OH}$ groups are also responsible for the agglomeration and compatibility of nanosilica particles in the host materials. Agglomeration is dependent on the high surface energy of the nanoparticles, and surface $\mathrm{OH}$ groups enhance this tendency through the formation of a large number of hydrogen bonds among the nanoparticles. Therefore, the removal of $\mathrm{OH}$ groups from the surface of nanosilica is a widely accepted approach for reducing agglomeration. Functionalisation is a common method for forming stable covalent bonds by replacing the $\mathrm{OH}$ groups using a coupling agent. However, it has been found that changes in the surface chemistry of nanoparticles will cause a chain reaction in the nanocomposite, which may not be in line with expectations. For example, nanosilica processed at a high temperature $\left(1050{ }^{\circ} \mathrm{C}\right.$ with dry nitrogen $)$ can effectively prevent moisture absorption, but it also forms larger and denser agglomerates than unprocessed nanosilica [14].

\section{Experimental Details}

\subsection{Preparation and Conditioning of Specimens}

The nanosilica powder was received from Sigma-Aldrich with a size range of 10-20 $\mathrm{nm}$. To reduce the amount of surface $\mathrm{OH}$ groups, trimethoxy(propyl)silane (C3) treatment was applied to the surface of the received nanosilica via an anhydrous route, and the related Fourier transform infrared results indicated that the propyl group was successfully bonded [4]. The methoxy group of the silane coupling agent reacts with the $\mathrm{OH}$ groups on the nanosilica, forming $\mathrm{Si}-\mathrm{O}-$ $\mathrm{Si}$ bonds attached to the propyl $\left(-\mathrm{C}_{3} \mathrm{H}_{7}\right)$ functional group. The nanocomposites incorporating untreated nanosilica will be referred to as ASR and those with C3-treated nanosilica as C3. The host polymer used was $20 \%$ high-density polyethylene (HDPE; Rigidex HD5813A, BP Chemical) blended with $80 \%$ LDPE (LD100BW, ExxonMobil Chemicals). The control group without nanosilica will be 
referred to as BPE. Four nanosilica loading ratios were used: $0.5,2,5$ and $10 \mathrm{wt} \%$. The nanocomposite materials were manufactured using a solvent method at a temperature of up to $160{ }^{\circ} \mathrm{C}[4,12]$. Film specimens were melting-pressed with a hydraulic press at $160{ }^{\circ} \mathrm{C}$. Once removed from the press, each specimen was placed directly into an oil bath at $115^{\circ} \mathrm{C}$ for 1 hour of isothermal crystallisation. To investigate the effect of differences in conditioning humidity on moisture absorption by the nanocomposites, all specimens were grouped and conditioned at room temperature under conditions of different relative humidity $(\mathrm{RH})$, including a vacuum desiccator (RH5), a climate room (RH60) and in deionised water (immersed condition) for about 9 days.

\subsection{Measurement of Moisture Absorption and TGA}

Moisture absorption by the nanocomposites was measured by the change in mass, taken as the average value of five specimens. In addition, a Perkin Elmer Pryis 1 TGA system was employed to perform TGA for untreated and C3-treated nanosilica particles that had already been conditioned in a humid environment (over RH90). The quantity of nanosilica tested was about $5 \mathrm{mg}$. The heating rate of TGA was $10{ }^{\circ} \mathrm{C} / \mathrm{min}$ in dry air over a test range from 50 to $900{ }^{\circ} \mathrm{C}$.

\subsection{Breakdown Strength}

DC breakdown measurements were conducted in silicone oil using spherical electrodes that were changed every 10 tests. The voltage ramping rate was $100 \mathrm{~V} / \mathrm{s}$. The specimen thickness of the nanocomposite films was $70 \pm 5 \mu \mathrm{m}$. The results obtained were analysed in terms of the Weibull distribution.

\subsection{Surface Potential Decay}

The schematic diagram of surface potential decay (SPD) was the same as in the previous study [12]. After negative corona charging, the first decay reading was taken after $5 \mathrm{~s}$ owing to system delay. In this work, the initial potential was set at 4.8 $\mathrm{kV}$. The corona charging lasted $180 \mathrm{~s}$, and was followed by a $900 \mathrm{~s}$ decay period. The thickness of the specimen was $120 \pm$ $5 \mu \mathrm{m}$.

\subsection{Space Charge}

The pulsed electro-acoustic (PEA) technique was employed for measuring space-charge behaviour. The equipment used was the same as in the previous study [17]. The thickness of the specimen was $120 \pm 5 \mu \mathrm{m}$. The space charge was tested for 2 hours.

\section{Results}

\subsection{Moisture Absorption and TGA}

The percentage change in mass for each specimen was calculated as

$M \%=\frac{M_{d}-M_{i}}{M_{i}} \times 100 \%$ ，

where $M_{d}$ is the mass measured after conditioning as a function of the number of days and $M_{i}$ is the initial mass of the specimen. The results are shown in Fig. 1. The changes in mass of specimens conditioned in RH5 did not changed, indicating that the greater increase in mass of the specimens conditioned at RH60 and Immersed was primarily due to direct contact with moisture. Therefore the results for specimens conditioned at RH5 are not shown here.
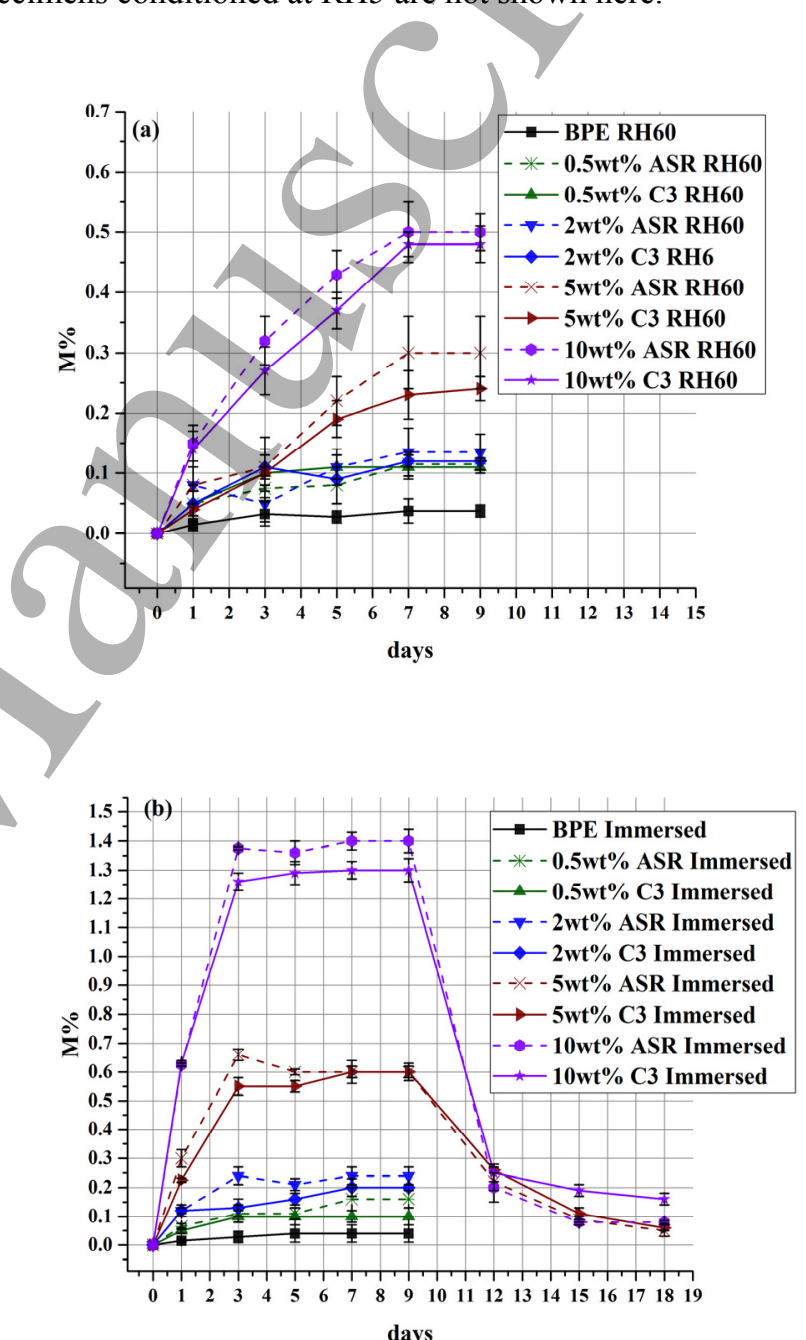

Fig. 1. Moisture absorption of nanocomposites conditioned (a) at RH60 or (b) by immersion in water.

The changes in mass of the specimens reached a steady state by 7 days. From Fig. 1 (a) and (b), it can be seen that the humidity of the conditioning environment directly affects the amount of moisture intake and the absorption rate, with a clear distinction between the RH60 and immersed specimens, especially for nanocomposites with high loading ratios (5 and $10 \mathrm{wt} \%)$. The increase in mass of the control, BPE, was 
much lower than that of the nanocomposites in both humid environments, which indicates that nanosilica is the main component causing moisture absorption in the nanocomposites. The amount and rate of moisture intake of nanocomposites with 5 and $10 \mathrm{wt} \%$ loading ratios are both greater than those of nanocomposites with 0.5 and $2 \mathrm{wt} \%$ loading ratios, since a higher proportion of nanosilica leads to a larger number of $\mathrm{OH}$ groups. Hence, apart from the environmental humidity, the loading ratio of nanosilica also has a significant effect on moisture absorption. Additionally, agglomerates with a high aspect ratio have frequently been reported in nanocomposites with loading ratios higher than 5 $w t \%[15,18]$. These high aspect ratios can further contribute to a significantly increased moisture intake. For the $\mathrm{C} 3$ nanocomposites, moisture absorption can still be seen, although the amount and rate are smaller than for the ASR nanocomposites. This indicates that the $\mathrm{OH}$ groups on the surface of nanosilica can be reduced in number by the $\mathrm{C} 3$ treatment, but not eliminated completely, and the residual $\mathrm{OH}$ groups are still able to absorb moisture via hydrogen bonding until a steady state is reached. It should be noted that in the case of the 5 and $10 \mathrm{wt} \%$ nanocomposites, the immersed specimens were replaced in the vacuum desiccator at room temperature after their conditioning in deionised water. As can be seen from Fig. 1(b), their masses were reduced sharply after this drying process.

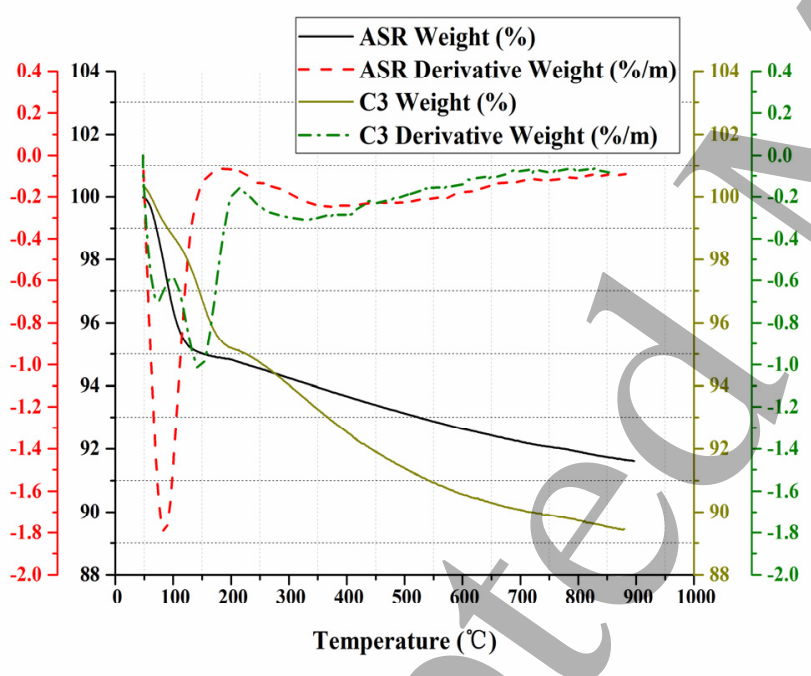

Fig. 2. TGA and DTG of ASR and C3 nanosilica.

The results of TGA and derivative thermogravimetric analysis (DTG) are shown for both ASR and C3 nanosilica particles in Figs. 2 and 3. Distinct DTG peaks can be seen from 50 to $200{ }^{\circ} \mathrm{C}$, which are attributed to loss of absorbed moisture from the surface of the nanosilica. In addition, there is another shallow but broad DTG peak from 200 to $500{ }^{\circ} \mathrm{C}$, mainly due to the $\mathrm{OH}$ groups on the nanosilica. The absorbed moisture contributes to the primary weight loss during the heating process. The TGA behaviour of the ASR nanosilica is different from that of the $\mathrm{C} 3$ nanosilica. For instance, from 50 to $200{ }^{\circ} \mathrm{C}$, the ASR nanosilica exhibits a sharp peak, whereas the $\mathrm{C} 3$ nanosilica exhibits two small peaks. As the C3 nanosilica is exposed longer to the humid environment, the first peak slowly comes to make up a large percentage of the weight loss, as shown in Fig. 3, which reveals that the $\mathrm{C} 3$ treatment can reduce moisture absorption compared with the ASR specimen. Furthermore, from 200 to $500{ }^{\circ} \mathrm{C}$ in Fig. 2, the $\mathrm{C} 3$ nanosilica loses more weight and does so even faster than the ASR nanosilica because it contains chemical groups that possess lower bond strength but higher molecular weight than $\mathrm{OH}$, such as carbon chains ( $\mathrm{C}-\mathrm{C}$ bonds).

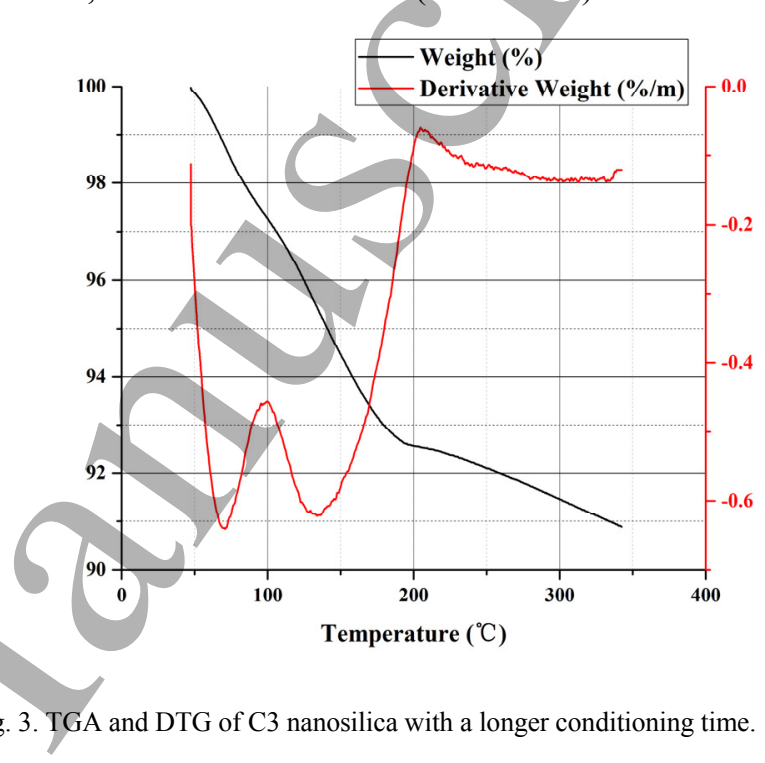

On heating from 50 to $900{ }^{\circ} \mathrm{C}$, the weight loss from the nanoparticles was about $11 \%$. Multiplying the loading ratios by this percentage gives values close to the changes in mass of the nanocomposites conditioned by immersion with the corresponding loading ratios. For example, the $M \%$ of $10 \mathrm{wt} \%$ nanocomposites is about $1.25 \%$, and that of $5 \mathrm{wt} \%$ nanocomposites is $0.55 \%$. In addition, according to the DTG curve, the majority of the weight loss occurred from 50 to $200{ }^{\circ} \mathrm{C}$. The absorbed moisture is mainly responsible for the change in mass of the nanocomposites, which is in line with the results of weighing.

\subsection{Breakdown Strength}

The results for DC breakdown strength as a function of nanosilica loading ratio and conditioning humidity are shown in Fig. 4. Twenty tests were conducted for each type of specimen and the results were processed according to a cumulative failure probability of $63.2 \%$ based on the Weibull distribution. The BPE control is represented by $0 \mathrm{wt} \%$. For the specimens conditioned at RH5, moisture absorption is not significant. An increase in loading ratio for both ASR and C3 nanosilica leads to a poorer DC breakdown strength regardless of how much moisture is absorbed by the 
specimen. In addition, Fig. 4 reveals that a rise in conditioning humidity can reduce the DC breakdown strength, but the reductions for BPE and for nanocomposites with $0.5 \mathrm{wt} \%$ loading ratio are not as sharp as for nanocomposites with 2,5 and $10 \mathrm{wt} \%$, which is consistent with the amount of absorbed moisture according to Fig. 1. The effect of C3 surface treatment also depends on the conditioning humidity and the loading ratio. For nanocomposites conditioned at $\mathrm{RH} 5, \mathrm{C} 3$ treatment is effective in mitigating the reduction of DC breakdown strength only for 2 and $5 \mathrm{wt} \%$ loading ratios, for those conditioned at RH60, it is effective for all loading ratios except $10 \mathrm{wt} \%$, and for those conditioned by immersion, it is effective only for a $0.5 \mathrm{wt} \%$ loading ratio. This indicates that the DC breakdown strength of the nanocomposites is dependent on both the species and amount of chemical groups on the surface of the nanosilica. Propyl groups are only effective for a certain range of nanosilica loading ratios, and they cannot completely prevent absorbed moisture from reducing DC breakdown strength when nanocomposites with higher loading ratios are exposed to a humid environment for a long time. The reason is that the remaining $\mathrm{OH}$ groups on the surface of the nanosilica can still absorb plentiful amounts of moisture. The difference between $10 \mathrm{wt} \%$ nanocomposites conditioned at RH60 and those conditioned by immersion is only about $10 \mathrm{kV} / \mathrm{mm}$. It appears that the amount of moisture absorbed by the $10 \mathrm{wt} \%$ nanocomposites conditioned at RH60 is sufficient to reduce the DC breakdown strength to its lowest value, and a $0.2 \mathrm{~mA}$ current was measured simultaneously with the breakdown.

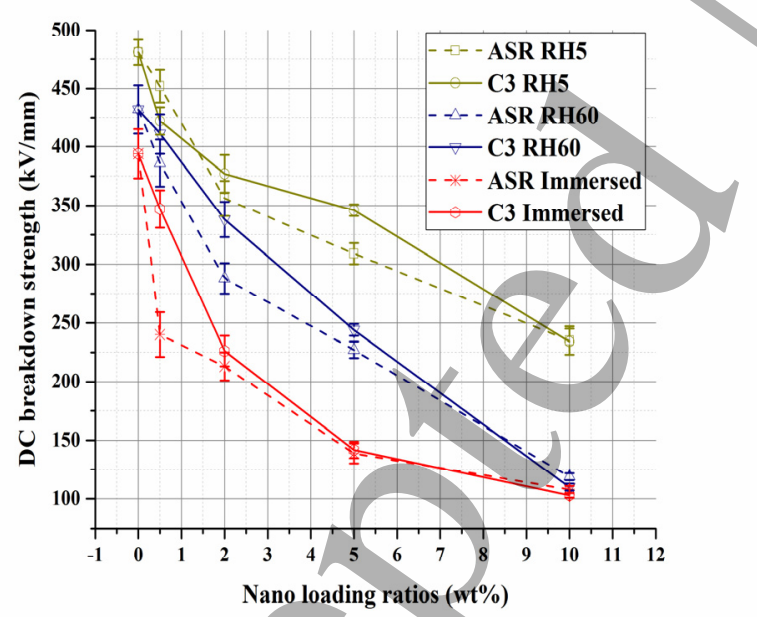

Fig. 4. DC breakdown strength of nanocomposites and BPE control (0 wt \%) as functions of nanosilica loading ratio and conditioning humidity.

To further investigate the impact of absorbed moisture on the DC breakdown strength, both the $5 \mathrm{wt} \%$ ASR and C3 specimens that were conditioned by immersion for 9 days were replaced in the vacuum desiccator (RH5) for re-drying at room temperature. Subsequently, DC breakdown was tested for these specimens. It can be seen from Fig. 5 that the breakdown strengths of both re-dried $5 \mathrm{wt} \%$ ASR and C3 specimens are improved compared with those of the immersed specimens. However, their breakdown strengths are still lower than those of the specimens simply conditioned at RH5. The DC breakdown strengths of the redried $5 \mathrm{wt} \%$ ASR and $\mathrm{C} 3$ specimens are similar. After immersion of specimens in deionised water and re-drying, the $\mathrm{C} 3$ treatment does not have the same effect as it does for the $5 \mathrm{wt} \%$ nanocomposites conditioned at RH5.

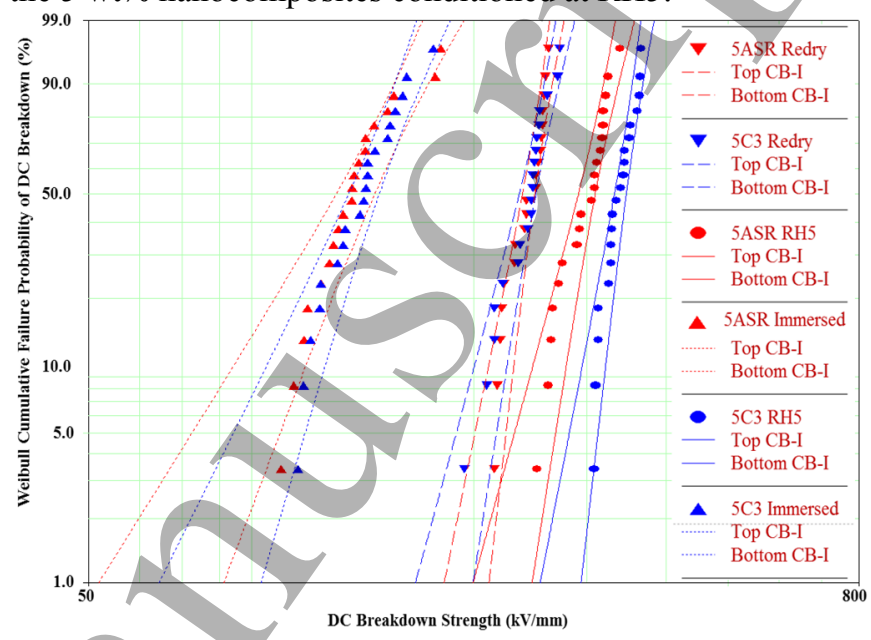

Fig. 5. Weibull plots comparing the DC breakdown strength of $5 \mathrm{wt} \%$ ASR and $\mathrm{C} 3$ nanocomposites conditioned at RH5, conditioned by immersion, or re-dried after conditioning by immersion.

\subsection{Surface Potential Decay}

To reveal the relationship between moisture absorption and the rate of decay of the surface potential, the normalised surface potential curve is presented. This shows the characteristics of surface potential decay for each type of specimen clearly and directly [19]. The normalisation procedure takes the surface potential $V_{t}$ at any time and divides it by the initially recorded surface potential $V_{\mathrm{o}}$ to give the normalized surface potential

$$
V_{t}^{*}=V_{t} / V_{0}
$$

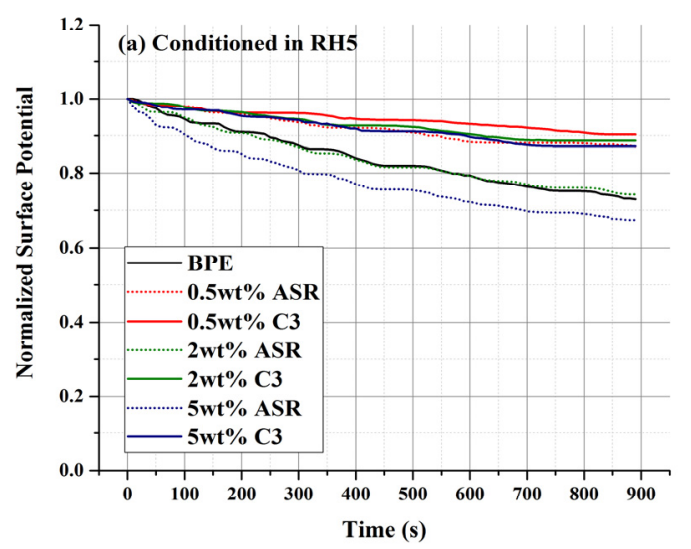



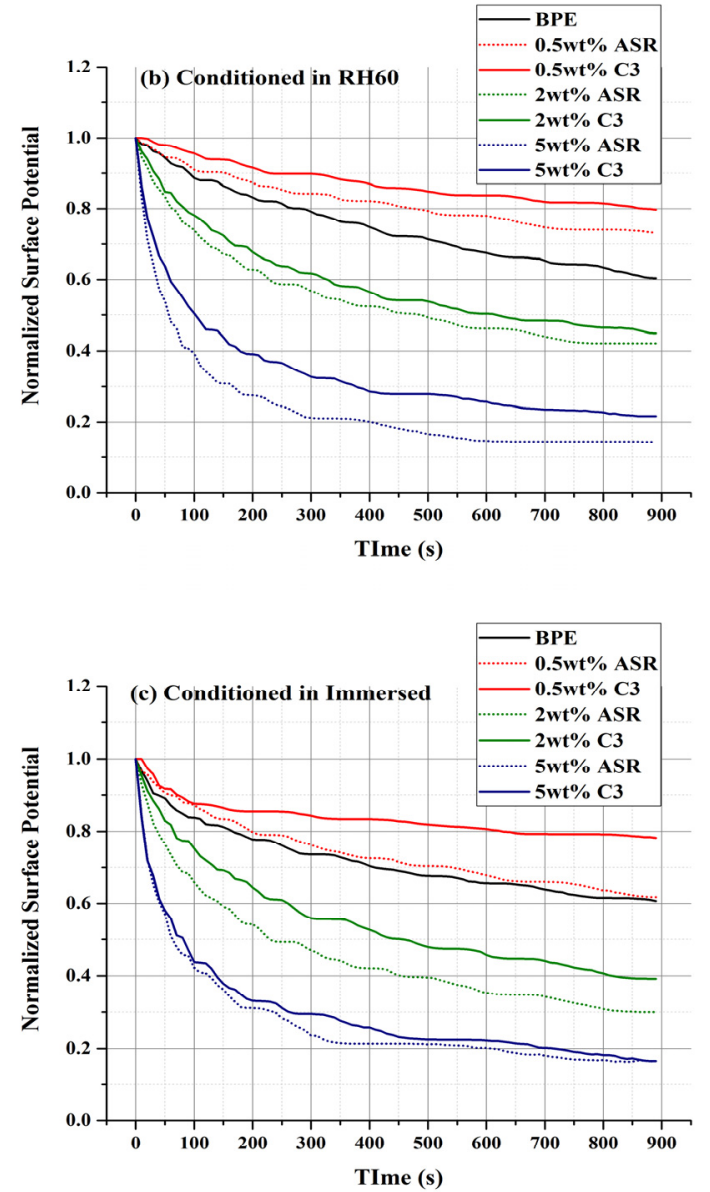

Fig. 6. Normalised surface potential decay of specimens conditioned (a) in a vacuum desiccator (RH5), (b) in a climate room (RH60), or (c) by immersion in water.

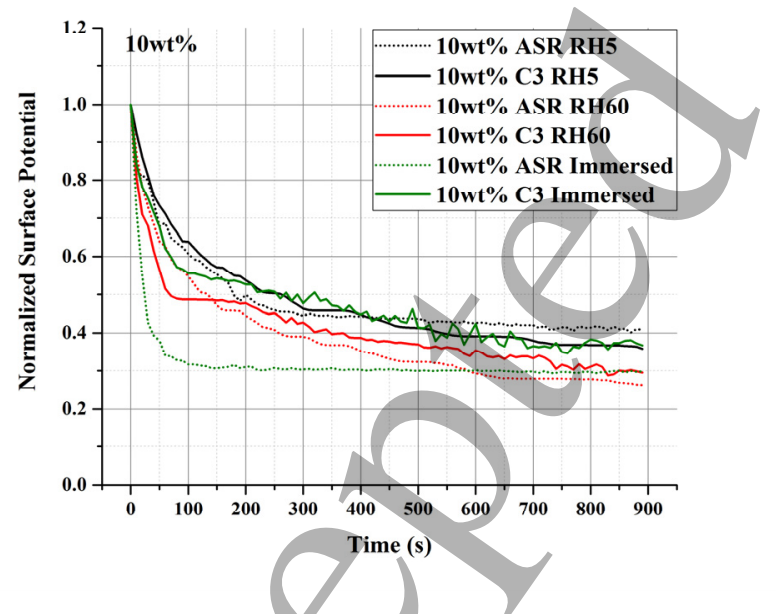

Fig. 7. Normalised surface potential decay of $10 \mathrm{wt} \%$ nanocomposites.

A steady rather than rapidly decreasing SPD curve usually means that charges cannot be easily transported through the specimen owing to the presence of deep traps. The results for a specimen conditioned at RH5 are shown in Fig. 6(a), from which it can be seen that the surface potential of the $0.5 \mathrm{wt} \%$ ASR decays more slowly than that of the BPE, and the decay rate clearly increases with increasing loading ratio. This is because the presence of nanosilica particles in the nanocomposite induces the formation of deep traps that capture charge carriers during charge transport [20]. However, at high nanosilica loading ratios, the distance between adjacent deep traps is small, and a tunnelling process becomes possible, enhancing charge transport in the nanocomposite [12,21]. The SPD results for the specimens conditioned at RH60 are presented in Fig. 6(b), which shows that absorbed moisture facilitates charge transport. As the results of weighing show, the $0.5 \mathrm{wt} \%$ nanocomposites are not able to absorb much moisture, which is good reason to suppose that the deep traps in the $0.5 \mathrm{wt} \%$ nanocomposites still have some effect on trapping charges and slowing charge transport in comparison with BPE. On the other hand, the 2 and $5 \mathrm{wt} \%$ nanocomposites show a sharp decay of surface potential compared with BPE, owing to the large amount of moisture that they are able to absorb. The results for the specimens conditioned in deionised water show comparable trends to those for the specimens conditioned at RH60, but the decay rates are greater, as illustrated in Fig. 6(c). The SPD of $10 \mathrm{wt} \%$ nanocomposites is different from that of specimens with other loading ratios. All the curves in Fig. 7 decrease dramatically within $100 \mathrm{~s}$, after which they reach a steady state or behave like noise. A reasonable explanation for this is the existence of a tunnelling effect in the $10 \mathrm{wt} \%$ nanocomposites, which is comparable to the effect of absorbed moisture in these specimens. Furthermore, if the SPD curve of BPE is taken as reference, the SPD results for specimens conditioned at RH60 or by immersion can be considered in terms of the nanosilica loading ratio. The surface potentials of the $\mathrm{C} 3$ nanocomposites decrease slightly more slowly than those of the ASR nanocomposites with the same loading ratio. $\mathrm{C} 3$ treatment can slow the decay for 2 and $5 \mathrm{wt} \%$ nanocomposites conditioned at RH5. However, for $10 \mathrm{wt} \%$ nanocomposites conditioned in a highhumidity environment, C3 treatment is not effective, which is in line with the DC breakdown results.

\subsection{Space Charge}

Measurement of space charge was undertaken for 2 hours with voltage poling. Specimens that absorbed moisture to the state of saturation had to be excluded owing to the likelihood of premature failure during the test. Therefore, the specimens used for space-charge measurement were conditioned at RH60 for just 1 day. From the space-charge profiles of $5 \mathrm{wt} \%$ ASR under a stress of $40 \mathrm{kV} / \mathrm{mm}$ shown in Fig. 8(a) and (b), it can be seen that the effect of absorbed moisture on the injection and transport of charge carriers depends strongly on the conditioning humidity. During the initial 2 minutes after charge injection, more homocharges are detected near the cathode for conditioning at RH60 than for conditioning at RH5. This may indicate that absorbed moisture can reduce the energy barrier between the specimen and the electrode. 
Thus, more charge can be injected into the specimen. The heterocharges gradually accumulate in both cases for up to 2 hours. However, the space charge accumulates more strongly in $5 \mathrm{wt} \%$ ASR conditioned at RH60 than in the same material conditioned at RH5. The positive charge observed near the cathode is transported from the anode, and recombination of positive and negative charges may occur during the transport process.
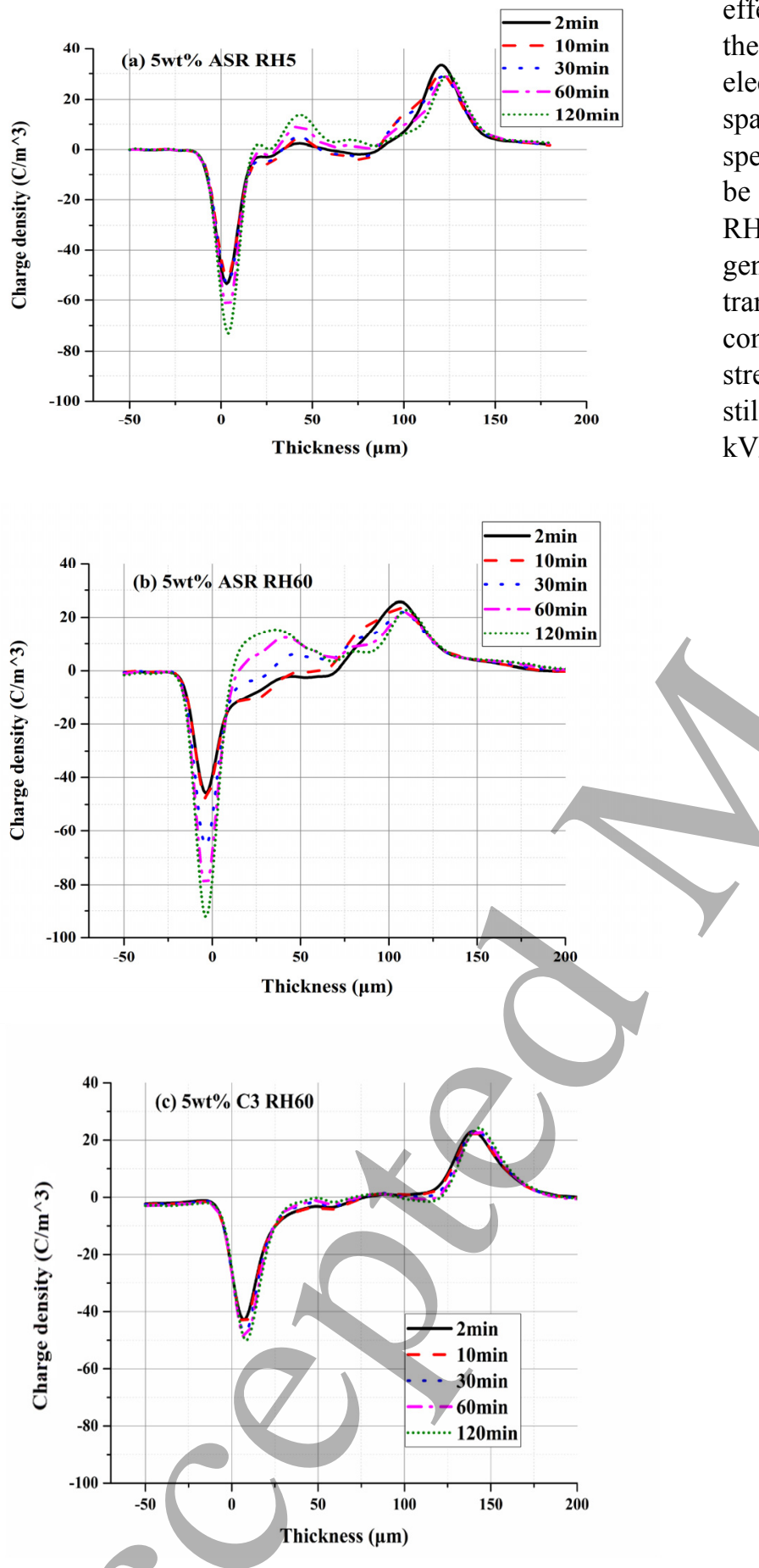

Fig. 8. Space-charge profiles of $5 \mathrm{wt} \%$ nanocomposites: (a) $5 \mathrm{wt} \%$ ASR conditioned at RH5; (b) $5 \mathrm{wt} \%$ ASR conditioned at RH60; (c) $5 \mathrm{wt} \% \mathrm{C} 3$ conditioned at RH60
From Fig. 8(c), it appears that space charge does not accumulate in the $5 \mathrm{wt} \% \mathrm{C} 3$ specimen conditioned at RH60. This behaviour can be explained as follows. As shown in Fig. 1 the amount and rate of moisture absorption in $\mathrm{C} 3$ nanocomposites are low compared with ASR nanocomposites, and so the moisture absorbed by $5 \mathrm{wt} \% \mathrm{C} 3$ does not have a significant effect on space-charge dynamics. In addition, the dried $5 \mathrm{wt} \% \mathrm{C} 3$ specimen is capable of effectively suppressing space-charge accumulation [17]. For the $0.5 \mathrm{wt} \%$ ASR nanocomposites presented in Fig. 9, the electric field stress is increased to $50 \mathrm{kV} / \mathrm{mm}$. However, space-charge injection is effectively suppressed in the specimen conditioned at RH5. Although a few charges can be observed near the anode of the specimen conditioned at RH60, there is no indication of further charge transport. In general, the high electric field enhances the injection and transport of charge carriers through the bulk. However, compared with the space-charge dynamics in $5 \mathrm{wt} \% \mathrm{ASR}$ stressed at $40 \mathrm{kV} / \mathrm{mm}, 0.5 \mathrm{wt} \%$ ASR conditioned at RH60 still possesses the ability to suppress space charge at 50 $\mathrm{kV} / \mathrm{mm}$, which is consistent with the results for SPD.
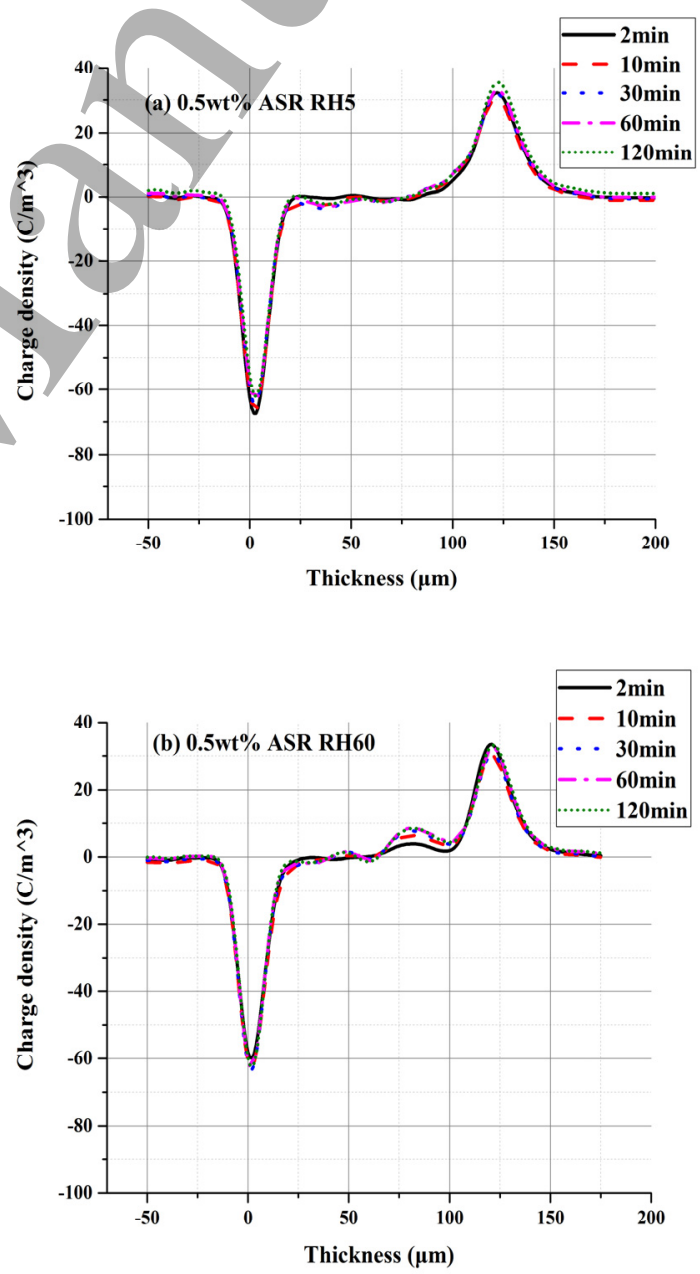

Fig. 9. Space-charge profiles of $0.5 \mathrm{wt} \%$ nanocomposites conditioned at (a) RH5 or (b) RH60. 


\section{Discussion}

The results presented above indicate that there is more than one form of absorbed moisture in the nanocomposites. It follows that previous explanations of moisture absorption in this context are inadequate. Further understanding can be obtained by application of the Zhuravlev model to the specific case of nanosilica. Although it has already been confirmed that moisture uptake is caused by the presence of surface $\mathrm{OH}$ groups on the nanosilica, the different effects of these groups and of moisture $\left(\mathrm{H}_{2} \mathrm{O}\right)$ on electrical properties are rarely mentioned. The $\mathrm{OH}$ groups are covalently bonded to the nanosilica surface, whereas the $\mathrm{H}_{2} \mathrm{O}$ molecules are physically bonded to the $\mathrm{OH}$ groups primarily via hydrogen bonds but also via van der Waals bonds [11]. Removal of moisture is called dehydration and removal of $\mathrm{OH}$ is called dehydroxylation [11]. The most common approach to the removal of $\mathrm{OH}$ groups from nanosilica is surface functionalisation $[17,22]$, while at least some of the absorbed moisture can be effectively removed by subjecting the nanocomposite to a vacuum even at room temperature [15]. According to the Zhuravlev model, moisture attached to the surface of nanosilica can be divided into two phases: multilayer moisture, which can be removed at $25{ }^{\circ} \mathrm{C}$ in vacuum, and monolayer moisture, which requires a temperature of $190{ }^{\circ} \mathrm{C}$ in vacuum for its removal [11]. The amount of monolayer moisture in a nanocomposite is limited by the amount of $\mathrm{OH}$ groups present on the nanosilica surface, because the monolayer is composed of $\mathrm{H}_{2} \mathrm{O}$ molecules physically bonded to these groups. On the other hand, the amount of multilayer moisture that is physically bonded with monolayer moisture or other multilayer moisture depends on the humidity and the conditioning time in addition to the amount of $\mathrm{OH}$ groups. The TGA results for wetted nanosilica in Figs. 2 and 3 indicate that the dehydroxylation of ASR nanosilica is a continuous process for both multilayer and monolayer moisture during the heating process. In contrast, for $\mathrm{C} 3$ nanosilica, the dehydroxylation is divided into two phases. The first peak, at about $75{ }^{\circ} \mathrm{C}$ represents desorption of the multilayer moisture. The second peak, at $150{ }^{\circ} \mathrm{C}$, represents desorption of monolayer moisture. Since the TGA was performed in dry air, the temperature differs slightly from that according to the Zhuravlev model, which performs in a vacuum condition. Although the temperature of moisture desorption can be obtained from experimental measurements, it is still difficult to distinguish between monolayer and multilayer moisture in a nanocomposite. This is mainly because of dispersion and agglomeration of nanosilica in the nanocomposite. Assuming that each $\mathrm{OH}$ group attached to the nanosilica surface absorbs one water molecule, the combined mass of these molecules is equal to the mass of the monolayer. The number of $\mathrm{OH}$ groups per unit surface area of silica is constant and is equal to $4.6 \mathrm{~nm}^{2}$ according to the least-squares method [11], and the surface area of the nanosilica is $175-225 \mathrm{~m}^{2} \mathrm{~g}^{-1}$ according to data from Sigma-Aldrich, and so the density of $\mathrm{OH}$ groups (i.e. the number of groups per unit mass of nanosilica) can be roughly calculated as $2.2 \times 10^{18}-$ $2.8 \times 10^{18} \mathrm{~g}^{-1}$. The mass of a water molecule is $3 \times 10^{-23} \mathrm{~g}$. The proportion of monolayers in the $10 \mathrm{wt} \%$ ASR nanocomposites can then be calculated as $2.4 \%$, which is larger than the value of $1.3 \%$ obtained from specimen weighing. It follows that only some of the $\mathrm{OH}$ groups are involved in moisture absorption. This is a result of agglomeration, which can prevent some parts of the silica surface from coming into contact with the moisture in the conditioning environment. However, as the change in mass of the nanocomposite reaches a steady state, the value obtained by weighing reflects the total absorbed moisture, most of which still represents multilayer moisture.

In general, polyethylene starts to melt above $110^{\circ} \mathrm{C}$ and the initial thermal aging temperature is about $90{ }^{\circ} \mathrm{C}$ [23]. Hence, there is no effective approach that can completely dehydrate a wetted specimen without causing some damage. In addition, total removal of the $\mathrm{OH}$ groups from the nanosilica surface would require a temperature of at least $900{ }^{\circ} \mathrm{C}$ [11]. Thus, the effect of the $\mathrm{OH}$ groups on the nanosilica surface should always be taken into consideration if no other process is applied to the nanocomposite. However, the specimens were manufactured at $160{ }^{\circ} \mathrm{C}$ with vigorous stirring, and this did allow removal of the majority of the moisture. Thus, the impact of monolayer and multilayer moisture/ on these specimens can be ignored for those conditioned at RH5. As the moisture absorption by a nanocomposite reaches a steady state, multilayer moisture is able to cover the surface of each nanosilica particle and indeed to overlap multiple particles. The results of surface potential decay and space charge measurements indicate that a more electrically conductive network will be formed by this overlapping multilayer moisture, allowing rapid charge transport through the specimens, especially for nanocomposites with loading ratios higher than $0.5 \mathrm{wt} \%$. C3 surface treatment partly replaces $\mathrm{OH}$ groups by propyl groups, inhibiting the formation of overlapping multilayer moisture. This can explain why charge dynamics, including injection, accumulation and transport, is suppressed in both wetted and dried C3 nanocomposites compared with ASR nanocomposites. Nevertheless, if the $\mathrm{C} 3$ specimens with high loading ratios are exposed to a humid environment for a sufficiently long time, the absorbed moisture will eventually come to dominate the nanosilica surface.

By considering the results for DC breakdown together with the Zhuravlev model, the most significant factor leading to a deterioration in breakdown strength can be determined. The amount of moisture absorbed depends on both the humidity of the environment and the nanosilica loading ratio. Moreover, the moisture absorbed by a nanocomposite can be 
divided into monolayer moisture and multilayer moisture, both of which contribute to a reduction in DC breakdown strength. From Fig. 4, it can be seen that increasing the loading ratios of both ASR and C3 nanocomposites leads rapidly to a decrease in breakdown strength. Based on the tunnelling model [21], it is believed that the main reason for the lower DC breakdown strength of nanocomposites with high loading ratios is the short separation between nanoparticles, which allows tunnelling to occur. However, the DC breakdown results show that the breakdown strength of $0.5 \mathrm{wt} \%$ nanocomposites is also slightly lower than that of $\mathrm{BPE}$. This can be explained by the ramping voltage used in the DC breakdown measurement. During the test, the electric field stress on the specimen increases as the applied voltage increases, and deep traps capture sufficient charges, which results in an increased local electric field, and a high electric field is an important factor allowing a large current flow through a specimen as a result of tunnelling. Thus, DC breakdown of $0.5 \mathrm{wt} \%$ nanocomposites, which possess a greater number of deep traps, can occur more easily than in BPE. According to the Zhuravlev model, the monolayer moisture cannot be removed by exposure to a vacuum at room temperature. Thus, the reduced breakdown strength of the re-dried specimen is due to the monolayer moisture. It can be assumed that only multilayer moisture is removed during re-drying and that the moisture retained in the specimen is monolayer moisture. For $5 \mathrm{wt} \% \mathrm{ASR}$, the changes in mass caused by multilayer and monolayer moisture are $0.5 \%$ and $0.05 \%$, respectively, as shown in Fig. 1. Correspondingly, the percentage reductions in DC breakdown strength caused by multilayer and monolayer moisture are $38 \%$ and $17 \%$ according to Fig. 5. For $5 \mathrm{wt} \%$ $\mathrm{C} 3$, the change in mass is similar to that for $5 \mathrm{wt} \% \mathrm{ASR}$. However, the percentage reductions in breakdown strength caused by multilayer and monolayer moisture are $31 \%$ and $28 \%$, respectively, which indicates that C3 treatment can mitigate the impact of multilayer moisture on DC breakdown strength by preventing this moisture from overlapping multiple nanosilica particles. Based on both ASR and C3 nanocomposites, the relative contributions of multilayer moisture to the reduction in DC breakdown strength is more obvious. However, it should be mentioned that the reduction in DC breakdown strength per mass of moisture absorbed in monolayer form is five times larger than that per mass of moisture absorbed in multilayer form. The main factors affecting moisture absorption are the loading ratio of nanosilica and the environmental humidity, and since extremely high levels of humidity are not frequently encountered in practice, it is the loading ratio that has the greatest role in reducing DC breakdown strength.

\section{Conclusions}

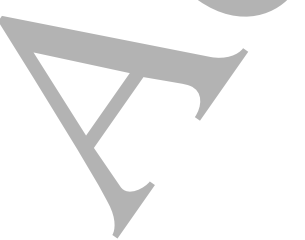

Experimental results on weight measurement, TGA, DC breakdown, SPD and space charge have revealed the impact of absorbed moisture on the breakdown strength and charge dynamics of polyethylene/nanosilica-based nanocomposites. The Zhuravlev model has been employed to identify the effects of $\mathrm{OH}$ groups and absorbed moisture on the nanosilica surface. The following conclusions can be drawn from the results of these investigations:

1. The amount and rate of moisture absorbed by the nanocomposites are proportional to the environmental humidity and the nanosilica loading ratio and are related to the number of $\mathrm{OH}$ groups present on the nanosilica surface.

2. Moisture absorbed by the nanocomposites leads to a reduction in DC breakdown strength and an enhancement of charge dynamics.

3. Compared with monolayer moisture, large amounts of multilayer moisture have a more significant impact on electrical properties and charge dynamics. However, the impact of multilayer moisture can be easily eliminated without destruction of the material. The amount of monolayer moisture per unit mass has a more significant impact on electrical properties than the amount of multilayer moisture.

4. C3 treatment cannot completely replace $\mathrm{OH}$ groups by propyl groups, and the remaining $\mathrm{OH}$ groups can still absorb moisture.

5. The loading ratio of nanosilica is the most significant factor causing a reduction in DC breakdown strength, followed by the amount of moisture absorbed.

\section{References}

[1]Lewis T 1994 IEEE Transactions on Dielectrics and Electrical Insulation 1)(5), 812 .

[2]Murakami Y, Nemoto M, Okuzumi S, Masuda S, Nagao M, Hozumi N, Sekiguchi Y, and Murata Y 2008 IEEE Transactions on Dielectrics and Electrical Insulation 15 (1).

[3]Fabiani D, Montanari G C, and Testa L 2010 IEEE Transactions on Dielectrics and Electrical Insulation 17 (1).

[4]Lau K, Vaughan A, Chen G, Hosier I, and Holt A 2013 Journal of Physics D: Applied Physics 46 (9), 095303.

[5]Hui L, Schadler L S, and Nelson J K 2013 IEEE Transactions on Dielectrics and Electrical Insulation 20 (2), 641.

[6]Du B, He Z, Du Q, and Guo Y 2016 IEEE Transactions on Dielectrics and Electrical Insulation 23 (1), 134.

[7]Momeni K, Odegard G, and Yassar R 2010 Journal of Applied Physics $108(11), 114303$

[8]Coleman J N, Khan U, and Gun'ko Y K 2006 Advanced materials 18 (6), 689.

[9]Momeni K 2014 Nano Energy 4, 132.

[10]Zou C, Fothergill J C, and Rowe S W 2008 IEEE Transactions on Dielectrics and Electrical Insulation 15 (1).

[11]Zhuravlev L 2000 Colloids and Surfaces A: Physicochemical and Engineering Aspects 173 (1), 1.

[12]Wang Y, Xu Z, Chen G, and Vaughan A Dielectrics (ICD), 2016 IEEE International Conference on.

[13]Qiang D, Yan W, Chen G, and Andritsch T 2016 IEEE Conference on Electrical Insulation and Dielectric Phenomena (CEIDP).

[14]Praeger M, Hosier I, Vaughan A, and Swingler S Electrical Insulation Conference (EIC), 2015 IEEE. 
[15]Hosier I, Praeger M, Vaughan A, and Swingler S Electrical Insulation Conference (EIC), 2015 IEEE.

[16]Yang J, Wang X, Zhao H, Zhang W, and Xu M 2014 IEEE Transactions on Dielectrics and Electrical Insulation 21 (4), 1957.

[17]Wang Y, Chen G, and Vaughan A Electrical Insulation and Dielectric Phenomena (CEIDP), 2014 IEEE Conference on.

[18]Yan W, Zhiqiang X, George C, and Vaughan A 2015 IEEE Conference on Electrical Insulation and Dielectric Phenomena (CEIDP).

[19]Shen W-W, Mu H-B, Zhang G-J, Deng J-B, and Tu D-M 2013 Journal of Applied Physics 113 (8), 083706.

[20]Wang Y, Hao M, Xu Z, Qiang D, Chen G, and Vaughan A 2018 Applied Physics Letters 113 (2), 022904.

[21]Chen G, Li S, and Zhong L 2015 IEEE 11th International Conference on the Properties and Applications of Dielectric Materials (ICPADM).

[22]Lau K, Vaughan A, Chen G, Hosier I, and Holt A Solid Dielectrics (ICSD), 2013 IEEE International Conference on.

[23]Li Z, Liu N, Gabriel S, and Chen G Electrical Insulation and Dielectric Phenomena (CEIDP), 2016 IEEE Conference on. 